\title{
Bidirectional treatment of peritoneal metastasis with Pressurized IntraPeritoneal Aerosol Chemotherapy (PIPAC) and systemic chemotherapy: a systematic review
}

\author{
Magnus Ploug ${ }^{*}$ (D), Martin Graversen, Per Pfeiffer and Michael Bau Mortensen
}

\begin{abstract}
Background: Pressurized intraperitoneal aerosol chemotherapy (PIPAC) is used in the palliative treatment of peritoneal metastasis. The combination of intraperitoneal and systemic chemotherapy seems rational, and the aim of this systematic review was to compare PIPAC directed monotherapy with a bidirectional treatment approach (PIPAC in combination with systemic chemotherapy). Main outcomes were survival and quality of life.

Methods: A systematic literature search in Medline, Embase, Cochrane and the "Pleura and Peritoneum" was conducted and analyzed according to PRISMA guidelines. Studies in English reporting on bidirectional treatment with PIPAC and systemic chemotherapy and published before April 2019 were included.

Results: Twelve studies with a total of 386 patients were included. None were specifically designed to compare mono- versus bidirectional treatment, but $44 \%$ of the patients received bidirectional treatment. This was more frequent in women (non-gynecological cancers) and one-third of the bidirectional treated patients had received no prior chemotherapy. Data from the included studies provided no conclusions regarding survival or quality of life.

Conclusion: Bidirectional treatment with PIPAC and systemic chemotherapy is practised and feasible, and some patients are enrolled having received no prior systemic chemotherapy for their PM.

The difficulty in drawing any conclusions based on this systematic review has highlighted the urgent need to improve and standardize reports on PIPAC directed therapy. We have, therefore, constructed a list of items to be considered when reporting on clinical PIPAC research.
\end{abstract}

Trial registration: International Prospective Register of Systematic Reviews, PROSPERO. Registration number: 90352, March 5, 2018.

Keywords: PIPAC, Bidirectional, Peritoneal metastasis, Intraperitoneal chemotherapy, Carcinomatosis

\footnotetext{
* Correspondence: Magnus.Ploug@rsyd.dk

Odense PIPAC Center (OPC) and Odense Pancreas Center (OPAC), Upper GI

and HPB Section, Department of Surgery, Odense University Hospital,

J.B.Winsløvs Vej 4, 5000 Odense C, Denmark
}

(c) The Author(s). 2020 Open Access This article is distributed under the terms of the Creative Commons Attribution 4.0 International License (http://creativecommons.org/licenses/by/4.0/), which permits unrestricted use, distribution, and reproduction in any medium, provided you give appropriate credit to the original author(s) and the source, provide a link to the Creative Commons license, and indicate if changes were made. The Creative Commons Public Domain Dedication waiver (http://creativecommons.org/publicdomain/zero/1.0/) applies to the data made available in this article, unless otherwise stated. 


\section{Background}

Pressurized intraperitoneal aerosol chemotherapy (PIPAC) is a novel approach in the palliative treatment of nonresectable peritoneal metastasis (PM) based on laparoscopically administered, aerosolized chemotherapy into the hyperbaric capnoperitoneum [1, 2]. Compared to systemic chemotherapy, PIPAC provides significantly higher concentrations of chemotherapeutics in the peritoneum, but a low concentration in the systemic circulation [3, 4] thereby, avoiding adverse effects from systemic administration. Since intravenous chemotherapy may enhance drug accumulation in the subperitoneal space and simultaneously have a direct effect on systemic micro-metastasis, the combination of intraperitoneal and systemic chemotherapy (i.e. bidirectional treatment) is a rational approach to treatment in patients with PM [4]. The bidirectional treatment approach might lead to a new mainstay in palliative cancer treatment, where the disseminated malignancy is targeted from two directions instead of one.

The idea of combining intraperitoneal and systemic chemotherapy is not new, and both treatments may be delivered in several different ways. Hyperthermic intraperitoneal chemotherapy (HIPEC) - introduced roughly 30 years before the first clinical PIPAC data [3, 5], is one method of delivering intraperitoneal chemotherapy (IP). Combined with cytoreductive surgery, HIPEC provides a potentially curative strategy in selected patients with PM $[6,7]$. Although trials on the use of PIPAC directed treatment as prophylaxis for intraperitoneal metastases are currently in progress, PIPAC is, to date, considered a palliative treatment. Through PIPAC, intraperitoneal chemotherapy can be delivered in a safe and repeatable way without systemic adverse effects. Objective local tumor response rates to PIPAC above $50 \%$ have been reported in patients with $\mathrm{PM}$ from a wide variety of primary cancers [8], without compromising the patient's quality of life (QoL) [8]. Bidirectional treatment strategies, not involving PIPAC, have been used and studied extensively and have shown improvement in overall and progression free survival compared to intraperitoneal treatment alone in patients with advanced ovarian or gastric cancers [9-12].

Based on these observations, a bidirectional approach combining PIPAC and systemic chemotherapy seems logical. Systemic chemotherapy, however, is associated with significant toxicity [13] and, a recent prospective multicenter study on patients with end-stage cancer, found that it did not improve QoL for patients with moderate and poor performance status. Furthermore, systemic chemotherapy had a significant deteriorating effect on QoL for patients with a good performance status [14]. In 2012, the American Society of Clinical Oncology advised against palliative chemotherapy for solid tumor malignancies in patients with poor performance status and no prior benefit from systemic treatment [15]. Hence, if systemic chemotherapy is introduced between PIPAC procedures, it is of great importance to monitor not only performance status and survival, but also QoL, complications and adverse events.

The primary aim of this systematic review was to investigate if bidirectional treatment with PIPAC plus systemic chemotherapy led to higher QoL scores and better survival than PIPAC alone in patients with PM of any origin. The secondary aim was to evaluate additional outcome variables in a bidirectional treatment setting including potential complications/adverse events and objective tumor response.

\section{Definition}

The term "bidirectional" in the delivery of chemotherapy is widely used but no clear or consensus definition exists [16-20]. Put simply, "bidirectional" means "operating or functioning in two (usually opposite) directions". With respect to chemotherapy, we define "bidirectional" as when two different administration routes are used in combination. Thus, in this review, bidirectional treatment describes when PIPAC is used in combination with systemic chemotherapy as opposed to monodirectional treatment where PIPAC is the only route of administration. In this definition, we included regimens where systemic chemotherapy was delivered either on the same day as PIPAC, between PIPAC procedures or in a continuous fashion during the PIPAC course (e.g. oral chemotherapy). We excluded treatment regimens where systemic chemotherapy was only given prior to the first PIPAC procedure, after the last PIPAC procedure or as a combination of the two strategies. In general, the term "bidirectional" should not be limited to a specific combination of administration routes but should rather be used to state that more than one route of administration is used in targeting the malignancy. This could be intravenous (IV) chemotherapy combined with IP (whether it is delivered as PIPAC, HIPEC, catheter-based or others) but also describes combinations including intraluminal chemotherapy or heretofore undiscovered ways of administering chemotherapy. Bidirectional chemotherapy, therefore, must always be accompanied by details on the routes of administration.

\section{Methods}

This review was performed according to the Preferred Reporting Items for Systematic Reviews and MetaAnalyses (PRISMA) guidelines [21]. A review protocol following the PRISMA-P guidelines [22] was published at the International Prospective Register of Systematic Reviews (PROSPERO - registration number: 90352) on March 5, 2018. Studies on adult patients with PM, written in English, and with reports of a bidirectional 
treatment regimen with PIPAC and systemic chemotherapy were considered for inclusion. No publication status restrictions were imposed, and only review articles and books/book chapters were excluded.

Studies were identified through a literature search in Medline (OVID interface, 1946-present), Embase (OVID interface, 1979-present) and in the Cochrane Central Register of Controlled Trials (CENTRAL). In addition, we manually searched the journal "Pleura and Peritoneum De Gruyter" (all issues to date) and scanned the reference lists of included studies or relevant systematic reviews.

To ensure literature saturation, an additional search was performed immediately prior to the submission of this review using the same search string but limited to prospective trials with the primary objective of comparing bidirectional to monodirectional PIPAC. To our knowledge, the first in-human application of intraperitoneal pressurized chemotherapy was performed in 2011 [3], and, therefore, our search was limited to the time period of 2011 to present. The specific search strategies were created in collaboration with a Health Sciences Librarian who has expertise in systematic review searching. We searched "Title and Abstracts" based on the following strategy (Medline search - Ovid interface)

1. PIPAC.ti,ab.

2. exp. Antineoplastic Agents/

3. chemotherap".ti,ab.

4. 2 or 3

5. (capnoperito" or pneumoperito* or pressur*).ti,ab.

6. (abdom* or intra-abdom* or intraabdom* or perito* or intraperito* or intra-perito*).ti,ab.

7. 4 and 5 and 6

8. 1 or 7

9. Limit 8 to (english language and humans and $\mathrm{yr}=$ "2011 -current")

The citations were uploaded to the COVIDENCE Software, (www.covidence.org), facilitating the selection process which was performed independently by two reviewers (MP and MG). In phase one of the selection process, titles and abstracts were screened and those that met the eligibility criteria (or where it was thought possible that a full text examination would find it to do so) were included. Subsequently, full text articles from all eligible works were examined, in detail. Disagreements between reviewers were resolved by consensus or, if consensus could not be achieved, a pre-designated third reviewer $(\mathrm{MBM})$ made the final decision on the inclusion of the article.

In order to evaluate the results after bidirectional therapy, overall survival (OS), progression free survival (PFS) and QoL were noted as main outcome variables. In addition, the performance status (PS) during inclusion, number of PIPAC procedures per patient, interval between PIPAC procedures, surgery related complications, adverse events and objective tumor response (OTR) were registered. Additionally, study details (author, title, publication year, study type), demographic information (age, gender, type of malignancy, time from diagnosis of PM to first PIPAC, previous treatment), intervention details (type and dosage of chemotherapy, ratio between mono- and bidirectional treated patients, frequency and duration of treatment) and finally any descriptive data with reasoning or comments on the bidirectional approach were included.

Data extraction was done independently by two reviewers (MP and MG) with disagreements resolved in the same manner as in the selection process.

If one of the reviewers suspected that data might be in duplicate, overlapping or reported in companion studies, this was evaluated by the group of authors. Publications from the same geographic location or where two or more authors were identical were examined for possible duplication. The risk of bias in individual studies and the risk of meta-bias(es) were also assessed.

\section{Results}

The primary literature search was conducted on March 6, 2018 (Fig. 1). The search of Medline, Embase, and The Cochrane Central Register of Controlled Trials yielded 616 citations while 52 citations were found in the journal "Pleura and Peritoneum". After adjusting for duplicates, 562 remained. 510 studies were excluded after reviewing the abstracts. The full text of the remaining 52 citations was examined and a further 38 citations were excluded, leaving 14 publications relevant for inclusion. No additional studies were found through the reference lists of included studies or from relevant systematic reviews. Upon careful examination of the 14 publications, two conference abstracts were found to possibly represent the same patients as reported in included articles. The abstract by Khomyakov et al. [23] was removed after contacting the author to confirm it represented the same patients as in their article [24]. The conference abstract by Robella et al. [25] was excluded due to convincing overlap in timeframe, author group and location with their included article [26], and due to the lack of any numerical data on bidirectional treatment. Thus, a total of 12 publications were included in the final analysis. The literature search was repeated on April 7th, 2019, but no additional studies were identified.

All publications were non-randomized and descriptive, having no control group and none were designed to compare bidirectional to monodirectional treatment.

There were 386 patients, in total, who were entered into a PIPAC program. Information on the distribution 


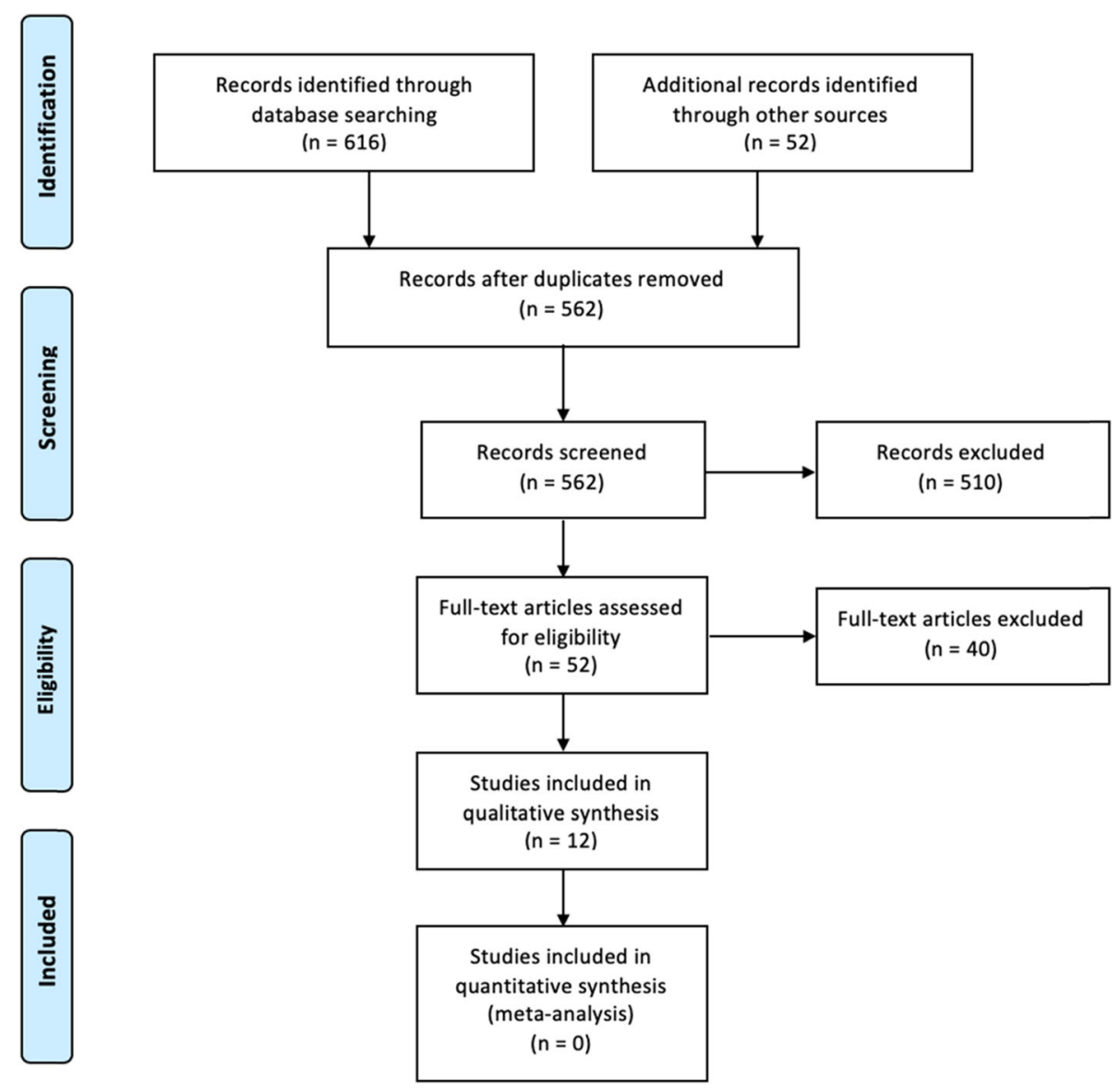

Fig. 1 Prisma flow chart [21]

between mono- and bidirectionally treated patients was available in 326 patients, with $44 \%$ receiving bidirectional treatment $(n=145)$ (Table 1$)$. Data on the type of malignancy in combination with the type of treatment (monovs. bidirectional) was reported in 211 patients and 38\% $(n=80)$ of these received bidirectional treatment with the following malignancy specific variations: gynecological $10 \%$ (9/87), pancreatic $27 \%$ (7/26), biliary tract $29 \%(4 / 14)$, mesothelioma 50\% (1/2), colorectal 68\% (13/19), gastric $74 \%$ (45/61), pseudomyxoma $100 \%(1 / 1)$ and cancer of unknown primary (CUP) $0 \%(0 / 1)$ (Table 1$)$.

From the available data on non-gynecological cancers we found that $50 \%$ of the men $(n=14 / 28)$ and $81 \%$ of the women $(n=25 / 31)$ received bidirectional treatment (Table 1).

Mean and median age ranged from 52 to 68 years. From four studies $[26,30,32,34]$ reporting raw data, the calculated median age was 62 years $(n=21$, interquartile range (IQR) 61-69) in the monodirectional group and 59 years $(\mathrm{n}=21$, IQR $51-68)$ in the bidirectional group (Table 1).

Performance status (PS), - either Eastern Cooperative Oncology Group (ECOG) or Karnofsky Index (KI), was reported as a criteria for entering the PIPAC program in six studies, and patients were excluded if PS(ECOG) was $>1$ [27], > 2 [24, 26, 30], > 3 [29], or if PS(KI) was $<50 \%[29,32]$. Two studies stated that poor PS was not an exclusion criterion [28, 33]. The actual PS was reported in five studies as a mean or median KI between 70 and $85 \%$ [28, 29, 31-33] with no separation between mono- and bidirectionally treated patients.

Previous tumor related surgery with primary tumor resection was performed in 145 out of 180 patients where data were available. Based on additional information in 143 of these patients 89\% ( $n=93 / 105)$ belonged to the monodirectional and $76 \%(n=29 / 38)$ to the bidirectional treatment group (Table 1).

Information on prior systemic chemotherapy was available in 10 publications, and 239 out of 284 patients (84\%) had received prior systemic chemotherapy when entering the PIPAC program. Not all publications specified this into mono- or bidirectional treated patients, but when this was specified, all monodirectional treated PIPAC patients $(n=105)$, but only two thirds of the patients receiving bidirectional therapy (44/69), had been pretreated with systemic chemotherapy (Table 1). The 


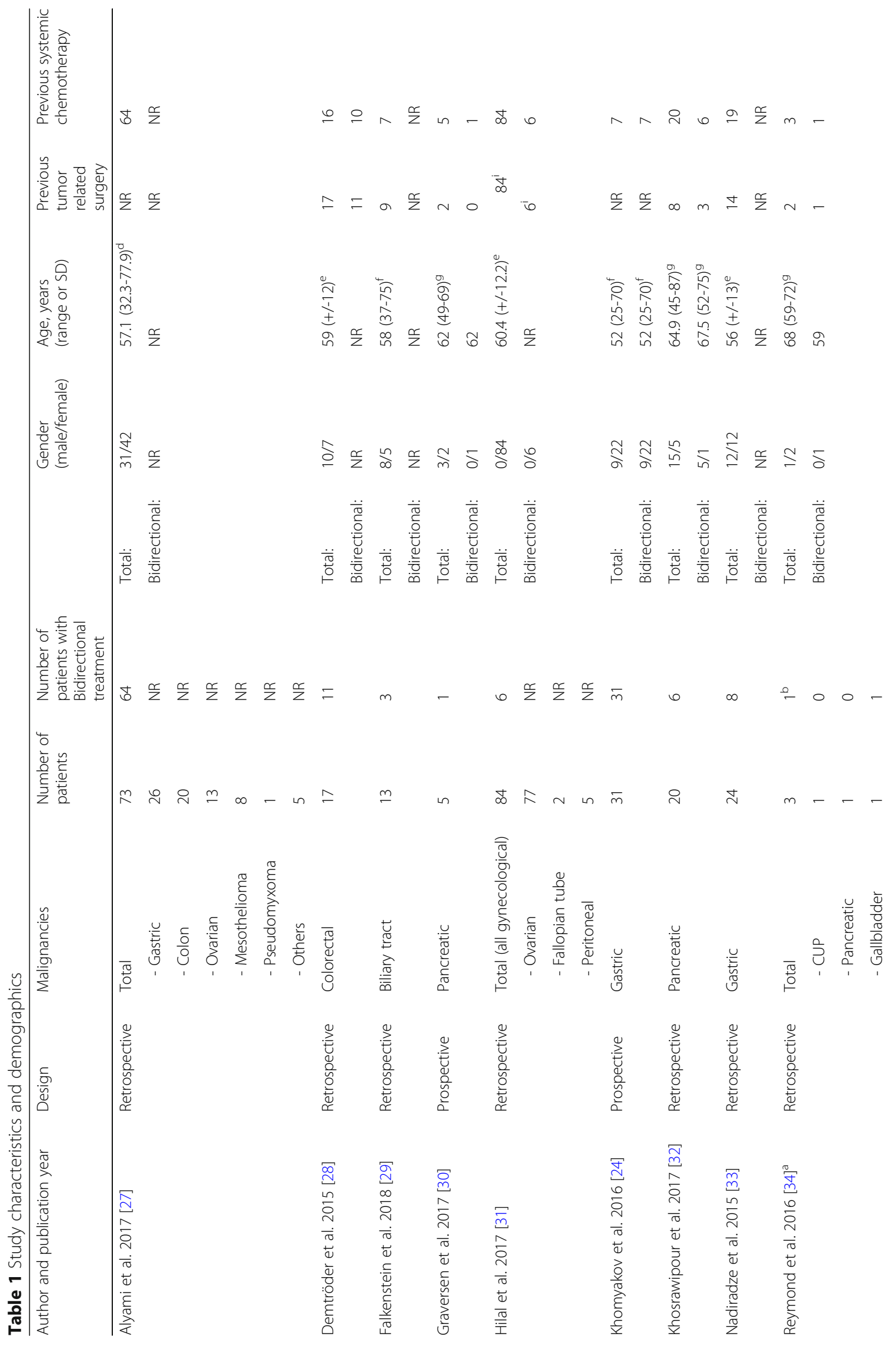




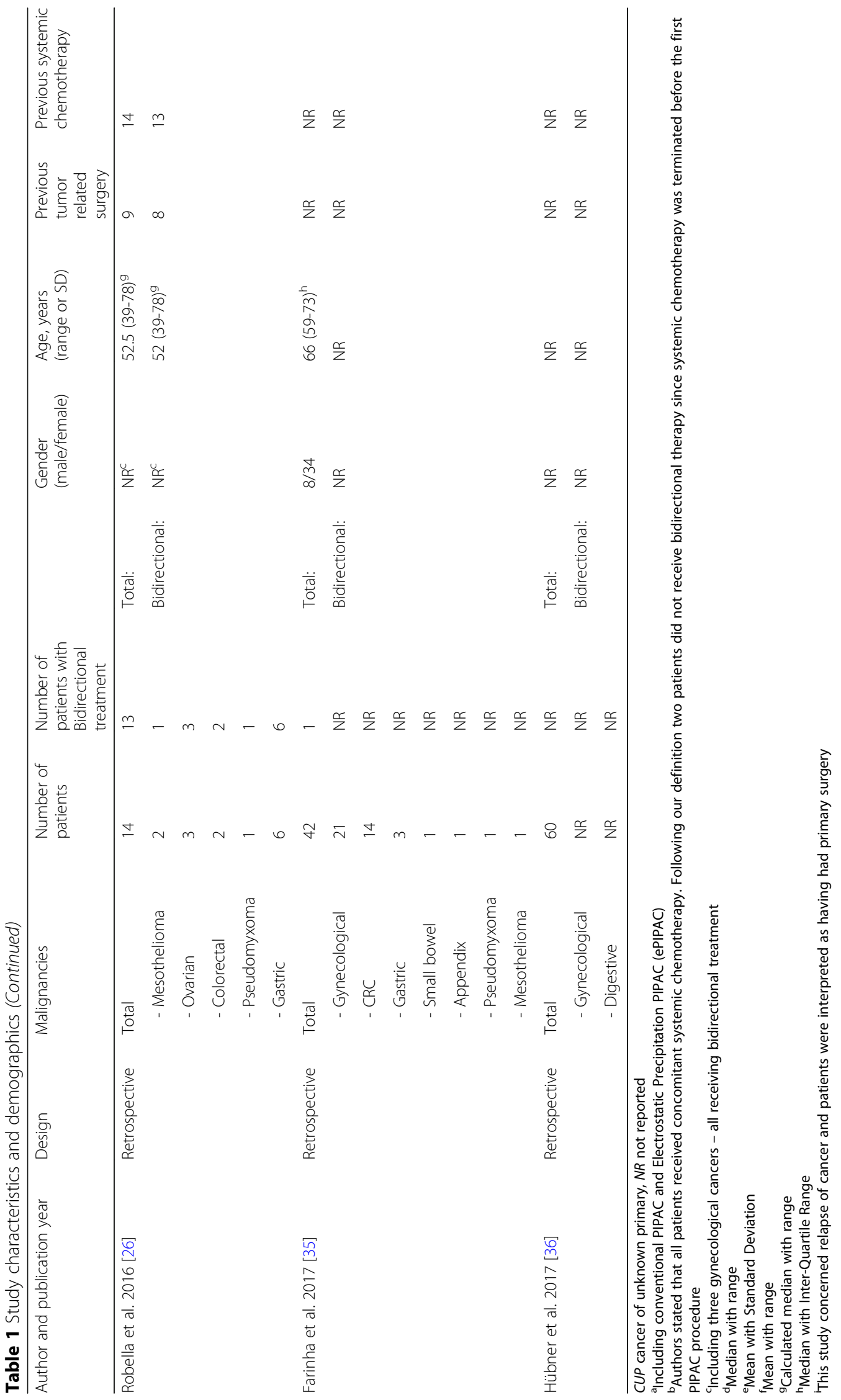


duration from diagnosis of PM to the first PIPAC treatment was noted in two trials, and reported as six and 2 months, respectively $[29,34]$.

The type of chemotherapy used for the PIPAC procedure was either Cisplatin/Doxorubicin or Oxaliplatin with one exception [27] where Mitomycin-c was used in six PIPAC procedures. Numerous different drugs were used for the systemic part of the bidirectional therapy, but with no clear description of the rationale behind their use [24, 26, 30, 32, 34]. Only two papers reported on the number of systemic chemotherapy cycles [30, 32]. The interval between PIPAC procedures was noted in 11 studies and reported to be either 6 weeks $[24,26$, $28,29,32-36]$ or four to six weeks [30, 31] (Table 2).

Overall survival, with a known starting point and with separate data on the mono- and bidirectional group, was only reported in eight patients (two treated bidirectionally) (Table 3).

QoL was reported in two studies (Table 3). One study [26] did not mention it in the methods section and wrote in the results section "QoL was recorded routinely in all patients before the enrolment and after each PIPAC-procedure through two questionnaires: SF-36 and EORTC QLQ-30. No further deterioration of physical, emotional and cognitive scores during therapy were recorded", but no data was presented. In the study by Farinha et al. [35], the main outcome was QoL assessed with the QLQ-C30 questionnaire but no comparison between mono- and bidirectional treated patients was made.

The median number of PIPAC procedures was 2 (IQR $1-3, n=200)$, calculated on raw data reported in nine studies (Table 4). The median number of PIPAC procedures in the bidirectional group was 2 (IQR $1-3, n=$ 52). Removing these 52 patients from the combined group the median number of PIPAC treatments remained 2 (IQR $1-3, n=148$ ) (Table 4 ).

Clavien Dindo (CD) classification was reported in two studies [30,35], with a timeframe of either 30-days [35] or not specified [30]. The results were limited to statements saying that "...adverse events and surgical complications were mild, transient and self-limiting" [30] and that the "overall complication rate was 8.8\%" [30].

Common Terminology Criteria for Adverse Events (CTCAE) data from 54 patients with information on treatment regimen (16 mono- and 38 bidirectional) showed that the proportion of patients experiencing an adverse event was $38 \%$ in the monodirectional and $21 \%$ in the bidirectional group - and these were almost exclusively grade 1-2 events (Table 4). Three studies

Table 2 Intervention details

\begin{tabular}{|c|c|c|c|c|}
\hline Author & Malignancies & PIPAC Chemo & Systemic Chemo & $\begin{array}{l}\text { Interval between } \\
\text { PIPAC }\end{array}$ \\
\hline Alyami et al. [27] & $\begin{array}{l}\text { Gastric, Colon, Ovarian, Mesothelioma, Pseudomyxoma } \\
\text { and others }\end{array}$ & Oxa, C/D or mito-c & NR & NR \\
\hline Demtröder et al. [28] & Colorectal & Oxa & NR & 6 weeks $^{a}$ \\
\hline Falkenstein et al. [29] & Biliary tract & $C / D$ & NR & 6 weeks $^{a}$ \\
\hline Graversen et al. [30] & Pancreatic & $C / D$ & $\mathrm{Gem}+\mathrm{S}-1$ & 4-6 weeks ${ }^{a}$ \\
\hline Hilal et al. [31] & Gynecological & $C / D$ & NR & 4-6 weeks ${ }^{a}$ \\
\hline Khomyakov et al. [24] & Gastric & $C / D$ & XELOX & 6 weeks $^{\mathrm{a}}$ \\
\hline Khosrawipour et al. [32] & Pancreatic & $C / D$ & $\begin{array}{l}\text { Gem+nab-Pax } \\
\text { Folfirinox } \\
\text { Gem }\end{array}$ & 6 weeks $^{\mathrm{a}}$ \\
\hline Nadiradze et al. [33] & Gastric & $C / D$ & NR & 6 weeks $^{a}$ \\
\hline Reymond et al. [34] ${ }^{a}$ & CUP, Pancreatic and Gallbladder & $C / D$ & $\mathrm{Cis}+\mathrm{Gem}$ & 6 weeks $^{a}$ \\
\hline Robella et al. [26] & $\begin{array}{l}\text { Mesothelioma, Ovarian, Colorectal, Pseudomyxoma } \\
\text { and Gastric }\end{array}$ & C/D or Oxa & $\begin{array}{l}\text { Topotecan } \\
\text { Folfox+cetuximab } \\
\text { Folfoxiri } \\
\text { Paclitaxel } \\
\text { Folfiri } \\
\text { Paclitaxel+Ramcirumab } \\
\text { Xelox } \\
\text { Paclitaxel } \\
\text { Pemetrexed }\end{array}$ & 6 weeks $^{a}$ \\
\hline Farinha et al. [35] & $\begin{array}{l}\text { Gynecological, Colorectal, Gastric, Small bowel, Appendix, } \\
\text { Pseudomyxoma and Mesothelioma }\end{array}$ & NR & NR & 6 weeks $^{a}$ \\
\hline Hübner et al. [36] & Gynecological and Digestive & C/D or OXA & NR & 6 weeks $^{a}$ \\
\hline
\end{tabular}


Table 3 Main outcome variables

\begin{tabular}{|c|c|c|c|c|}
\hline Author & Reported overall survival (yes/no) & $\begin{array}{l}\text { Separately reported overall survival on } \\
\text { mono- and bidirectional treated patients } \\
\text { yes/no (number of patients) }\end{array}$ & PFS reported (yes/no) & QoL reported (yes/no) \\
\hline Alyami et al. [27] & No & No & No & No \\
\hline Demtröder et al. [28] & Yes (from 1st PIPAC) & No & No & No \\
\hline Falkenstein et al. [29] & Yes (from 1st PIPAC) & No & No & No \\
\hline Graversen et al. [30] & Yes (from 1st PIPAC) & Yes $(n=5)$ & No & No \\
\hline Hilal et al. [31] & No & No & No & No \\
\hline Khomyakov et al. [24] & Yes (not clear from when) & Yes $(n=31)^{c}$ & No & No \\
\hline Khosrawipour et al. [32] & Yes (from 1st PIPAC) & No & No & No \\
\hline Nadiradze et al. [33] & Yes (from 1st PIPAC) & No & No & No \\
\hline Reymond et al. [34] ${ }^{a}$ & Yes (from 1st PIPAC) ${ }^{\mathrm{b}}$ & Yes $(n=3)$ & No & No \\
\hline Robella et al. [26] & No & No & No & Yes \\
\hline Farinha et al. [35] & No & No & No & Yes \\
\hline Hübner et al. [36] & No & No & No & No \\
\hline
\end{tabular}

PFS progression free survival, QoL Quality of life

aSurvival also reported from the time of primary tumor resection, and from the time of the PM diagnosis

${ }^{b}$ Survival also reported from the time of diagnosis (of either the primary cancer or of PM)

${ }^{\mathrm{c}}$ All patients treated bidirectionally

reported a substantial number of CTCAE grade $\geq 3$, but no information on the distribution between mono- and bidirectional treatment was provided [27, 28, 33].

OTR was reported using PCI, PRGS, TRG, RECIST or an unspecified histological regression model (Table 4). Four studies $[24,30,32,34]$ provided separate data on the bidirectional group, but these data were not homogenous enough to allow reporting and interpretation, and most of the patients received less than three PIPAC procedures before evaluation.

No included studies qualified for the evaluation of risk of bias in individual studies. The two prospective trials $[24,30]$ were evaluated for "outcome reporting bias" and found not to refer in their method section to a protocol or a clinical trial registration. Despite screening the International Clinical Trials Registry Platform (ICTRP), we were not able to match these studies to a protocol.

During screening for "positive publication bias", we searched for unpublished trials through the ICTRP and the ISRTCN registries, using the search terms "PIPAC", "pressurized chemotherapy" and "pressurised chemotherapy". The search was carried out on the 6th of February 2019. Nineteen studies were identified through ICTRP and two through ISRTCN. One study on bidirectional treatment was identified, investigating repetitive ePIPAC and simultaneous IV chemotherapy for colorectal PM. The trial (EudraCT: 2017-000927-29) is still running and, therefore, could not be included in this review. Additionally, when screening the journal "Pleura and Peritoneum", we identified two published protocols on ongoing PIPAC trials specifically evaluating the bidirectional approach in PM from upper GI- [37] (EudraCT:
2018-001035-40) and gastric cancer [38] (No registration number available).

No duplicate data were suspected among the included trials.

\section{Discussion}

This systematic review shows that the bidirectional treatment approach is practiced and feasible but that no studies have attempted to compare it to either monodirectional PIPAC or to monodirectional systemic treatment. All studies were descriptive and showed a high degree of variation in terms of which outcomes were reported and how the outcomes were described. The studies were very heterogeneous including multiple malignancy types, different time-points of the diseases, varying disease extent and varying degrees of pretreatment. Taken together, we were not able to draw any conclusions or perform a meta-analysis.

Regarding our main outcomes (OS, PFS and QoL), no comparisons between mono- and bidirectional treatment were possible - not even as simple additive analyses or narrative comments. Although 7 studies reported the overall survival using a similar definition (from the time of the first PIPAC procedure, practically no specific data on mono- vs. bidirectional treatment was published. PFS was not reported in any studies, while QoL data were reported in two studies, of which, only one provided actual data.

We chose the three outcomes that, in our view, are most important in assessing the potential benefits and harms of a bidirectional approach. A possible survival benefit due to the bidirectional treatment and to the 
Table 4 Additional outcome variables

\begin{tabular}{|c|c|c|c|c|c|c|c|c|}
\hline Author & $\begin{array}{l}\text { Median number of } \\
\text { PIPAC procedures } \\
\text { (number of patients) }\end{array}$ & $\begin{array}{l}\text { Median number } \\
\text { of PIPAC procedures } \\
\text { in bidirectional } \\
\text { treated patients }\end{array}$ & CTCAE Reporting & Total & Mono & Bidirectional & $\begin{array}{l}\text { Objective } \\
\text { tumor } \\
\text { response } \\
\text { reported }\end{array}$ & $\begin{array}{l}\text { Scale used } \\
\text { to report } \\
\text { objective } \\
\text { tumor response }\end{array}$ \\
\hline \multirow[t]{5}{*}{ Alyami et al. [27] } & \multirow[t]{5}{*}{$2(n=73)^{a}$} & \multirow[t]{5}{*}{$N R$} & CTCAE reported & Yes $^{c}$ & \multirow[t]{5}{*}{ No } & \multirow[t]{5}{*}{ No } & \multirow[t]{5}{*}{ Yes } & \multirow[t]{5}{*}{$\mathrm{PCl}$} \\
\hline & & & Time frame (days) & 30 & & & & \\
\hline & & & Grade 1-2 & NR & & & & \\
\hline & & & Grade 3-4 & 16 & & & & \\
\hline & & & Grade 5 & 5 & & & & \\
\hline \multirow[t]{5}{*}{ Demtröder et al. [28] } & \multirow[t]{5}{*}{$3(n=17)^{a}$} & \multirow[t]{5}{*}{ NR } & CTCAE reported & Yes $^{\mathrm{d}}$ & \multirow[t]{5}{*}{ No } & \multirow[t]{5}{*}{ No } & \multirow[t]{5}{*}{ Yes } & \multirow[t]{5}{*}{ TRG } \\
\hline & & & Time frame (days) & NR & & & & \\
\hline & & & Grade 1-2 & 12 & & & & \\
\hline & & & Grade 3-4 & 4 & & & & \\
\hline & & & Grade 5 & 0 & & & & \\
\hline \multirow[t]{5}{*}{ Falkenstein et al. [29] } & \multirow[t]{5}{*}{$1(n=13)^{a, b}$} & \multirow[t]{5}{*}{ NR } & CTCAE reported & Yes $^{d}$ & \multirow[t]{5}{*}{ No } & \multirow[t]{5}{*}{ No } & \multirow[t]{5}{*}{ Yes } & \multirow[t]{5}{*}{ TRG, PCl } \\
\hline & & & Time frame (days) & NR & & & & \\
\hline & & & Grade 1-2 & 9 & & & & \\
\hline & & & Grade 3-4 & 0 & & & & \\
\hline & & & Grade 5 & 0 & & & & \\
\hline \multirow[t]{5}{*}{ Graversen et al. [30] } & \multirow[t]{5}{*}{$3(n=5)^{a}$} & \multirow[t]{5}{*}{$3(n=1)^{a}$} & CTCAE reported & Yes & No & No & Yes & PRGS, RECIST \\
\hline & & & Time frame (days) & NR & & & & \\
\hline & & & Grade 1-2 & NR & & & & \\
\hline & & & Grade 3-4 & NR & & & & \\
\hline & & & Grade 5 & 0 & & & & \\
\hline Hilal et al. [31] & NR & NR & CTCAE reported & No & No & No & No & NR \\
\hline Khomyakov et al. [24] & $1(n=31)^{\mathrm{a}}$ & $1(n=31)^{\mathrm{a}}$ & CTCAE reported & Yes $^{d}$ & - & Yes & Yes & PRGS \\
\hline & & & Time frame (days) & 30 & & 30 & & \\
\hline & & & Grade 1-2 & 3 & & 3 & & \\
\hline & & & Grade 3-4 & 1 & & 1 & & \\
\hline & & & Grade 5 & 0 & & 0 & & \\
\hline Khosrawipour et al. [32] & $1.5(n=20)^{a, b}$ & $1(n=6)^{\mathrm{a}}$ & CTCAE reported & $Y_{e s}^{d}$ & Yes & Yes & Yes & TRG, PCl \\
\hline & & & Time frame (days) & NR & NR & NR & & \\
\hline & & & Grade 1-2 & 6 & 3 & 3 & & \\
\hline & & & Grade 3-4 & 0 & 0 & 0 & & \\
\hline & & & Grade 5 & 1 & 1 & 0 & & \\
\hline Nadiradze et al. [33] & $2(n=24)^{a}$ & NR & CTCAE reported & Yes $^{d}$ & No & No & Yes & Unspecified \\
\hline & & & Time frame (days) & NR & & & & $\begin{array}{l}\text { histological } \\
\text { regression }\end{array}$ \\
\hline & & & Grade 1-2 & 15 & & & & \\
\hline & & & Grade 3-4 & 7 & & & & \\
\hline & & & Grade 5 & 2 & & & & \\
\hline Reymond et al. [34] ${ }^{a}$ & $4(3)^{a}$ & $7(n=1)^{a}$ & CTCAE reported & Yes $^{d}$ & Yes & Yes & Yes & PRGS, RECIST \\
\hline & & & Time frame (days) & NR & NR & NR & & \\
\hline & & & Grade 1-2 & 3 & 2 & 1 & & \\
\hline & & & Grade 3-4 & 0 & 0 & 0 & & \\
\hline & & & Grade 5 & 0 & 0 & 0 & & \\
\hline
\end{tabular}


Table 4 Additional outcome variables (Continued)

\begin{tabular}{|c|c|c|c|c|c|c|c|c|}
\hline Author & $\begin{array}{l}\text { Median number of } \\
\text { PIPAC procedures } \\
\text { (number of patients) }\end{array}$ & $\begin{array}{l}\text { Median number } \\
\text { of PIPAC procedures } \\
\text { in bidirectional } \\
\text { treated patients }\end{array}$ & CTCAE Reporting & Total & Mono & Bidirectional & $\begin{array}{l}\text { Objective } \\
\text { tumor } \\
\text { response } \\
\text { reported }\end{array}$ & $\begin{array}{l}\text { Scale used } \\
\text { to report } \\
\text { objective } \\
\text { tumor response }\end{array}$ \\
\hline \multirow[t]{5}{*}{ Robella et al. [26] } & \multirow[t]{5}{*}{$3(n=14)^{a}$} & \multirow[t]{5}{*}{$3(n=13)^{a}$} & CTCAE reported & Yes $^{d}$ & \multirow[t]{5}{*}{ No } & \multirow[t]{5}{*}{ No } & \multirow[t]{5}{*}{ No } & \multirow[t]{5}{*}{$N R$} \\
\hline & & & Time frame (days) & $N R$ & & & & \\
\hline & & & Grade 1-2 & 14 & & & & \\
\hline & & & Grade 3-4 & 0 & & & & \\
\hline & & & Grade 5 & 0 & & & & \\
\hline Farinha et al. [35] & $N R$ & $N R$ & CTCAE reported & No & No & No & No & $N R$ \\
\hline Hübner et al. [36] & NR & NR & CTCAE reported & No & No & No & No & NR \\
\hline
\end{tabular}

CTCAE Common Terminology Criteria for Adverse Events, NR not reported, PCI Peritoneal Cancer Index, RECIST Response Evaluation Criteria in Solid Tumors, PRGS Peritoneal Regression Grading Score, TRG Tumor Regression Grading

a median calculated from presented data

${ }^{b}$ mean reported in this publication

creported per procedure

${ }^{d}$ reported per patient

presumable earlier initiation of PIPAC must be evaluated alongside its positive or negative impact on QoL. Our recommendation is that these outcomes should be standardized and included in any future PIPAC reporting.

We found a large variation in the reported PS criteria for patients entering PIPAC programs, but we were not able to evaluate PS specifically in the context of patients receiving bidirectional treatment. The median number of PIPAC procedures was similar in both the bidirectional and in the overall group, whereas the CTCAE reported adverse event rate was, surprisingly, lower in the bidirectional group. Theoretically, combining a surgical procedure with intravenous chemotherapy could lead to an increase in adverse events, thus, leading to a reduced number of PIPAC procedures - none of which were found. The data must be interpreted with great caution and unintentional selection of patients with good performance status for bidirectional treatment is a possible source of bias.

The reporting on complications and adverse events varied considerably across studies. One study [30] reported on surgical complications using Clavien-Dindo and on adverse events using CTCAE [30], while the remaining used either Clavien-Dindo [35] or CTCAE. When using CTCAE, eight of nine studies described this as a tool to report adverse events, but four of the studies then used the term "complications" in their CTCAE reporting in the results section. Another study [26] described that they used CTCAE to assess postoperative complications. Thus, it seems that the terms "adverse events" and "complications" are used interchangeably. Evaluation on whether an event is related to surgery (for which the Clavien-Dindo is intended [39]) or to the chemotherapeutic agent (where CTCAE is normally used) may prove difficult in a bidirectional treatment strategy. In the closely related field of CRS and HIPEC, Lehmann et al. showed that the interpretation of the severity of events between the two classifications differed with significantly higher major morbidity rates found using CTCAE compared to the Clavien-Dindo classification [40] - indicating that comparison between the two systems is not appropriate. Previous systematic reviews concluded that PIPAC directed therapy is associated with a low risk of complications and adverse events [1], but, in this review, data from the largest study with bidirectionally treated patients observed a significantly higher mortality and major complications rate than previously reported [27]. Despite its retrospective design it is important to note that these experienced PM centers had a learning curve regarding patient selection during the implementation of a PIPAC program, and that mortality and major complications occurred at the beginning of their experience. This observation was done in a mainly bi-directionally treated patient cohort and suggest that training in patient selection might lower the rate of adverse events.

OTR was reported using different definitions, and the majority of data were not specifically related to monoor bidirectional treatment results. We recommend the components and use of OTR should be defined for future comparative trials.

We observed more women than men receiving bidirectional treatment in non-gynecological cancers, and the rate of bidirectional treatment varied between 10 and $100 \%$ in different cancer types. The latter variation may be influenced by multi-modal treatment regimens being more common in some cancer diseases, and the fact that larger PIPAC data are still limited on several indications. One third of the patients receiving bidirectional therapy did so without prior systemic chemotherapy. Considering the lack of evidence of efficacy and safety, on bidirectional PIPAC, this approach may seem premature. The 
use of PIPAC directed therapy as an early intervention in PM is the focus of several new studies, and a reduction in time from diagnosis of PM to the initiation of PIPAC may improve survival data.

The above findings should be interpreted with caution. The risk of possible biases is considerable since the studies were not designed to compare mono- with bidirectional treatment, the number of patients was small, and the method of reporting was not uniform. Secondly, a substantial part of the data on bidirectional patients came from one publication [24] treating all patients this way, and comparison with monodirectional patients was done across publications. Outcome reporting bias is also possible since no published protocol or registration in a clinical trial registry was found regarding the two prospective trials. We did not identify any unpublished trials concerning bidirectional PIPAC, and therefore, no positive publication bias was identified. The difficulty in drawing any conclusions based on this systematic review of the literature has highlighted the urgent need to improve and standardize reports on PIPAC directed therapy. We have, therefore, constructed a list of items to be considered when reporting on clinical PIPAC research (Additional file 1).

When examining the included studies, additional comments on bidirectional treatment were recorded in a non-formalized manner. The treatment-free window (no systemic chemotherapy) was scheduled to be between one and four weeks before PIPAC $[26,28,31]$, and between zero and two weeks after the PIPAC procedure [26-28]. While the latter time frame is not relevant (using the present drugs for PIPAC), the use of some systemic drugs may prolong the treatment free interval in order to allow hematologic factors to normalize. The reported treatment intervals, however, and the need for a treatment-free window during bidirectional therapy are not based on scientific evidence. The bidirectional approach was performed on patient demand [28] and was, even though advised against by the PIPAC facility, independently sought by patients at outside institutions [31], indicating a patient-directed need for research on this treatment strategy. Bidirectional treatment was seen as an option to treat patients with PM and limited extraperitoneal disease, in which PIPAC as monotherapy was not indicated $[26,36]$. Some studies interpreted their own data or referred to data from other institutions as promising evidence regarding the bidirectional approach $[24,28,29,32]$. Some stated that PIPAC should enhance the efficacy of systemic therapy by reducing the intratumoural interstitial fluid pressure but without referencing this theory $[26,28]$. Based on data in this review and the PIPAC literature, in general, the scientific evidence behind some of the statements is limited, and there is a risk of continuous citing of undocumented statements.

\section{Conclusion}

Bidirectional treatment is practiced in many PIPAC centers and some patients are enrolled having received no prior systemic chemotherapy for their PM. Based on this systematic review we were unable to make any major conclusions stating whether bidirectional therapy is better or worse than PIPAC monotherapy. There is an urgent need for prospective trials focusing on bidirectional therapy and for consensus in determining how, when and which specific outcome variables should be reported.

We hope that this study, and the proposed list of items, will serves as a modest, but potential useful tool to help researchers improve international consensus on PIPAC reporting.

\section{Supplementary information}

Supplementary information accompanies this paper at https://doi.org/10. 1186/s12885-020-6572-6.

Additional file 1. Reporting items for PIPAC. List of items to be considered when reporting on PIPAC directed therapy.

\section{Abbreviations \\ C/D : Cisplatin/Doxorubicin; CD: Clavien Dindo; CENTRAL: Cochrane Central Register of Controlled Trials; CTCAE: Common Terminology Criteria for Adverse Events; CUP: Cancer of Unknown Primary; ECOG : Eastern Cooperative Oncology Group; ePIPAC: Electrostatic Precipitation PIPAC; gem : Gemcitabine; HIPEC: Hyperthermic Intraperitoneal Chemotherapy; ICTRP: International Clinical Trials Registry Platform; IP: Intraperitoneal chemotherapy; IQR: Inter-Quartile range; IV: Intravenous; KI: Karnofsky Index; mito-c: Mitomycin c; nab-pax: Nab-Paclitaxel; NR: Not Reported; OS : Overall Survival; OTR: Objective Tumor Response; OXA : Oxaliplatin; PCI: Peritoneal Cancer Index; PFS: Progression Free Survival; PIPAC: Pressurized Intra- Peritoneal Aerosolized Chemotherapy; PM: Peritoneal Metastasis; PRGS: Peritoneal Regression Grading Score; PRISMA : Preferred Reporting Items for Systematic Reviews and Meta-Analyses; PROSPERO: International Prospective Register of Systematic Reviews; PS: Performance Status; QoL: Quality of Life; RECIST: Response Evaluation Criteria in Solid Tumors; TRG: Tumor Regression Grading}

\section{Acknowledgements}

Proofreading: Gordon McSheffrey, MD, MSC.

Paediatric Subspecialty Fellow, University of Toronto.

\section{Authors' contributions}

The following author has made a substantial contribution. MP: To the conception and design of the work and to the acquisition, analysis and interpretation of data, and have drafted the work. MG: To the conception and design of the work and to the acquisition and interpretation of data and have substantively revised the work. PF: To the interpretation of data and have substantively revised the work. MBM: To the conception and design of the work and to the interpretation of data and have substantively revised the work. All authors read and approved the final manuscript.

\section{Funding}

None.

Availability of data and materials

All data generated or analyzed during this study are included in this published article.

Ethics approval and consent to participate Not applicable. 


\section{Consent for publication}

Not applicable.

\section{Competing interests}

The authors declare that they have no competing interests.

Received: 10 June 2019 Accepted: 23 January 2020

Published online: 10 February 2020

\section{References}

1. Grass F, Vuagniaux A, Teixeira-Farinha H, Lehmann K, Demartines N, Hübner $M$, et al. Systematic review of pressurized intraperitoneal aerosol chemotherapy for the treatment of advanced peritoneal carcinomatosis. $\mathrm{Br}$ J Surg. 2017;104:669-78.

2. Khosrawipour V, Khosrawipour T, Kern AJP, Osma A, Kabakci B, Diaz-Carballo $D$, et al. Distribution pattern and penetration depth of doxorubicin after pressurized intraperitoneal aerosol chemotherapy (PIPAC) in a postmortem swine model. J Cancer Res Clin Oncol. 2016;142:2275-80.

3. Solass W, Kerb R, Murdter T, Giger-Pabst U, Strumberg D, Tempfer C, et al. Intraperitoneal chemotherapy of peritoneal carcinomatosis using pressurized aerosol as an alternative to liquid solution: first evidence for efficacy. Ann Surg Oncol. 2014;21:553-9.

4. de Bree E, Michelakis D, Stamatiou D, Romanos J, Zoras O. Pharmacological principles of intraperitoneal and bidirectional chemotherapy. Pleura Peritoneum. 2017:2:47-62.

5. Neuwirth MG, Alexander HR, Karakousis GC. Then and now: cytoreductive surgery with hyperthermic intraperitoneal chemotherapy (HIPEC), a historical perspective. J Gastrointest Oncol. 2016;7:18-28.

6. Verwaal VJ, van Ruth S, de Bree E, van Sloothen GW, van Tinteren $H$, Boot H, et al. Randomized trial of cytoreduction and hyperthermic intraperitoneal chemotherapy versus systemic chemotherapy and palliative surgery in patients with peritoneal carcinomatosis of colorectal cancer. J Clin Oncol. 2003;21:3737-43.

7. van Driel WJ, Koole SN, Sikorska K, Schagen van Leeuwen JH, HWR S, RHM $\mathrm{H}$, et al. Hyperthermic Intraperitoneal chemotherapy in ovarian Cancer. N Engl J Med. 2018:378:230-40.

8. Graversen M, Detlefsen S, Bjerregaard JK, Fristrup CW, Pfeiffer P, Mortensen MB. Prospective, single-center implementation and response evaluation of pressurized intraperitoneal aerosol chemotherapy (PIPAC) for peritoneal metastasis. Ther Adv Med Oncol. 2018;10:175883591877703.

9. Kitayama J, Ishigami H, Yamaguchi H, Sakuma Y, Horie H, Hosoya Y, et al. Treatment of patients with peritoneal metastases from gastric cancer. Ann Gastroenterol Surg. 2018;2:116-23.

10. Markman M, Bundy BN, Alberts DS, Fowler JM, Clark-Pearson DL, Carson LF, et al. Phase III trial of standard-dose intravenous cisplatin plus paclitaxel versus moderately high-dose carboplatin followed by intravenous paclitaxel and intraperitoneal cisplatin in small-volume stage III ovarian carcinoma: an intergroup study of the oncology group, southwestern oncology group, and eastern cooperative oncology group. J Clin Oncol. 2001;19:1001-7.

11. Armstrong DK, Bundy B, Wenzel L, Huang HQ, Baergen R, Lele S, et al. Intraperitoneal Cisplatin and paclitaxel in ovarian Cancer. NEJM. 2006;354: $34-43$.

12. Alberts D, Liu P, Dward HAnnigan EV, Robert O, Willliams S, Ames Y, Oung $J A$, et al. Intraperitoneal cisplatin plus intravenous cyclophosphamide versus intravenous cisplatin plus intravenous cyclophosphamide for stage III ovarian cancer. NEJM. 1996;335:1950-5.

13. Schuurhuizen CSEW, Braamse AMJ, Konings IRHM, Sprangers MAG, Ket JCF, Dekker J, et al. Does severe toxicity affect global quality of life in patients with metastatic colorectal cancer during palliative systemic treatment? A systematic review. Ann Oncol. 2017;28:478-86.

14. Prigerson HG, Bao Y, Shah MA, Elizabeth Paulk M, LeBlanc TW, Schneider BJ, et al. Chemotherapy use, performance status, and quality of life at the end of life. JAMA Oncol. 2015;1(6):778-84.

15. Schnipper LE, Smith TJ, Raghavan D, Blayney DW, Ganz PA, Mulvey TM, et al. American Society of Clinical Oncology identifies five key opportunities to improve care and reduce costs: the top five list for oncology. J Clin Oncol Off J Am Soc Clin Oncol. 2012;30:1715-24.

16. Yonemura Y, Canbay E, Li Y, Coccolini F, Glehen O, Sugarbaker PH, et al. A comprehensive treatment for peritoneal metastases from gastric cancer with curative intent. Eur J Surg Oncol. 2016;42:1123-31.
17. Le Roy F, Gelli M, Hollebecque A, Honoré C, Boige V, Dartigues P, et al. Conversion to complete cytoreductive surgery and hyperthermic intraperitoneal chemotherapy for malignant peritoneal mesothelioma after bidirectional chemotherapy. Ann Surg Oncol. 2017;24:3640-6.

18. Mehta AM, Huitema ADR, Burger JWA, Brandt-Kerkhof ARM, Van Den Heuvel SF, Verwaal VJ. Standard clinical protocol for bidirectional hyperthermic intraperitoneal chemotherapy (HIPEC): systemic leucovorin, 5-fluorouracil, and heated intraperitoneal oxaliplatin in a chloride-containing carrier solution. Ann Surg Oncol. 2017;24:990-7.

19. Bijelic L, Stuart OA, Sugarbaker P. Adjuvant bidirectional chemotherapy with intraperitoneal pemetrexed combined with intravenous cisplatin for diffuse malignant peritoneal mesothelioma. Gastroenterol Res Pract. 2012;2012: 890450. https://doi.org/10.1155/2012/890450.

20. Valle SJ, Alzahrani NA, Liauw W, Sugarbaker PH, Bhatt A, Morris DL. Hyperthermic intraperitoneal chemotherapy (HIPEC) methodology, drugs and bidirectional chemotherapy. Indian J Surg Oncol. 2016;7:152-9.

21. Moher D, Liberati A, Tetzlaff J, Altman DG. Preferred reporting items for systematic reviews and meta-analyses: the PRISMA statement. Int I Surg. 2010;8:336-41.

22. Moher D, Shamseer L, Clarke M, Ghersi D, Liberati A, Petticrew M, et al. Preferred reporting items for systematic review and meta-analysis protocols (PRISMA-P) 2015 statement. Syst Rev. 2015;4:1-1.

23. Khomyakov V, Ryabov A, Bolotina L, Ivanov A, Cheremisov V, Kolobaev I, et al. Initial experience of pressurized intraperitoneal aerosol chemotherapy (PIPAC) for treatment of peritoneally disseminated gastric cancer. Ann Oncol. 2016;27 Suppl 2:1-85.

24. Khomyakov V, Ryabov A, Ivanov A, Bolotina L, Utkina A, Volchenko N, et al. Bidirectional chemotherapy in gastric cancer with peritoneal metastasis combining intravenous XELOX with intraperitoneal chemotherapy with low-dose cisplatin and doxorubicin administered as a pressurized aerosol: an open-label, Phase-2 study (PIPAC-GA2). Pleura Peritoneum. 2016;1:159-66.

25. Robella M, Vaira M. Pressurized intraperitoneal aerosol chemotherapy (PIPAC) associated with systemic chemotherapy: an innovative approach for peritoneal carcinomatosis. Eur J Surg Oncol. 2016;42:S155.

26. Robella $\mathrm{M}$, Vaira $\mathrm{M}$, De Simone $\mathrm{M}$. Safety and feasibility of pressurized intraperitoneal aerosol chemotherapy (PIPAC) associated with systemic chemotherapy: an innovative approach to treat peritoneal carcinomatosis. World J Surg Oncol. 2016;14:128.

27. Alyami M, Gagniere J, Sgarbura O, Cabelguenne D, Villeneuve L, Pezet D, et al. Multicentric initial experience with the use of the pressurized intraperitoneal aerosol chemotherapy (PIPAC) in the management of unresectable peritoneal carcinomatosis. Eur J Surg Oncol. 2017;43:2178-83.

28. Demtroder C, Solass W, Zieren J, Strumberg D, Giger-Pabst U, Reymond M$A A$, et al. Pressurized intraperitoneal aerosol chemotherapy with oxaliplatin in colorectal peritoneal metastasis. Color Dis. 2016;18:364-71.

29. Falkenstein TA, Gotze TO, Ouaissi M, Tempfer CB, Giger-Pabst U, Demtroder $C$, et al. First clinical data of pressurized Intraperitoneal aerosol chemotherapy (PIPAC) as salvage therapy for peritoneal metastatic biliary tract cancer. Anticancer Res. 2018;38:373-8.

30. Graversen M, Detlefsen SS, Bjerregaard JK, Pfeiffer P, Mortensen MB. Peritoneal metastasis from pancreatic cancer treated with pressurized intraperitoneal aerosol chemotherapy (PIPAC). Clin Exp Metastasis. 2017;34:309-14.

31. Hilal Z, Rezniczek GAGA, Klenke R, Dogan A, Tempfer CB, et al. Nutritional status, cachexia, and anorexia in women with peritoneal metastasis and intraperitoneal chemotherapy: A longitudinal analysis. J Gynecol Oncol. 2017:28:1-10.

32. Khosrawipour T, Khosrawipour V, Giger-Pabst U. Pressurized intra peritoneal aerosol chemotherapy in patients suffering from peritoneal carcinomatosis of pancreatic adenocarcinoma. PLoS One. 2017;12:1-11.

33. Nadiradze G, Giger-Pabst U, Zieren J, Strumberg D, Solass W, Reymond MAA. Pressurized Intraperitoneal aerosol chemotherapy (PIPAC) with low-dose Cisplatin and doxorubicin in gastric peritoneal metastasis. J Gastrointest Surg. 2016;20:367-73.

34. Reymond M, Demtroeder C, Solass W, Winnekendonk G, Tempfer C. Electrostatic precipitation pressurized IntraPeritoneal aerosol chemotherapy (ePIPAC): first in-human application. Pleura Peritoneum. 2016;1:109-16.

35. Teixeira Farinha H, Grass F, Kefleyesus A, Achtari C, Romain B, Montemurro $M$, et al. Impact of pressurized Intraperitoneal aerosol chemotherapy on quality of life and symptoms in patients with peritoneal carcinomatosis: a retrospective cohort study. Gastroenterol Res Pract. 2017;4596176:1-10. 
36. Hubner M, Grass F, Teixeira-Farinha H, Pache B, Mathevet P, Demartines N, et al. Pressurized IntraPeritoneal aerosol chemotherapy - practical aspects. Eur J Surg Oncol. 2017;43:1102-9.

37. Oliver Goetze T, Al-Batran S-E, Pabst U, Reymond M, Tempfer C, Bechstein WO, et al. Pressurized intraperitoneal aerosol chemotherapy (PIPAC) in combination with standard of care chemotherapy in primarily untreated chemo naïve upper gi-adenocarcinomas with peritoneal seeding - a phase II/III trial of the AIO/CAOGI/ACO. Pleura Peritoneum. 2018. https://doi.org/10. 1515/pp-2018-0113.

38. Eveno C, Jouvin I, Pocard M. PIPAC EstoK 01: pressurized IntraPeritoneal aerosol chemotherapy with cisplatin and doxorubicin (PIPAC C/D) in gastric peritoneal metastasis: a randomized and multicenter phase II study. Pleura Peritoneum. 2018. https://doi.org/10.1515/pp-2018-0116.

39. Dindo D, Demartines N, Clavien P-A. Classification of surgical complications: a new proposal with evaluation in a cohort of 6336 patients and results of a survey. Ann Surg. 2004;240:205-13.

40. Lehmann K, Eshmuminov D, Slankamenac K, Kranzbühler B, Clavien P-A, Vonlanthen $\mathrm{R}$, et al. Where oncologic and surgical complication scoring systems collide: time for a new consensus for CRS/HIPEC. World I Surg. 2016:40:1075-81.

\section{Publisher's Note}

Springer Nature remains neutral with regard to jurisdictional claims in published maps and institutional affiliations.

Ready to submit your research? Choose BMC and benefit from:

- fast, convenient online submission

- thorough peer review by experienced researchers in your field

- rapid publication on acceptance

- support for research data, including large and complex data types

- gold Open Access which fosters wider collaboration and increased citations

- maximum visibility for your research: over $100 \mathrm{M}$ website views per year

At BMC, research is always in progress.

Learn more biomedcentral.com/submissions 